\title{
Expression and distribution of PPP2R5C gene in leukemia
}

\author{
Haitao Zheng ${ }^{1 \dagger}$, Yu Chen ${ }^{1,2 \dagger}$, Shaohua Chen ${ }^{1}$, Yuzhe Niu ${ }^{1,2}$, Lijian Yang ${ }^{1}$, Bo Li ${ }^{1}$, Yuhong Lu', Suxia Geng ${ }^{3}$, Xin Du ${ }^{3}$ \\ and Yangqiu $\mathrm{Li}^{1,2^{*}}$
}

\begin{abstract}
Background: Recently, we clarified at the molecular level novel chromosomal translocation $t(14 ; 14)(q 11 ; q 32)$ in a case of Sézary syndrome, which caused a rearrangement from TRAJ7 to the PPP2R5C gene. PPP2R5C is one of the regulatory B subunits of protein phosphatase 2A (PP2A). It plays a crucial role in cell proliferation, differentiation, and transformation. To characterize the expression and distribution of five different transcript variants of the PPP2R5C gene in leukemia, we analyzed the expression level of PPP2R5C in peripheral blood mononuclear cells from 77 patients with de novo leukemia, 26 patients with leukemia in complete remission (CR), and 20 healthy individuals by real-time PCR and identified the different variants of PPP2R5C by RT-PCR.

Findings: Significantly higher expression of PPP2R5C was found in AML, CML, T-ALL, and B-CLL groups in comparison with healthy controls. High expression of PPP2R5C was detected in the B-ALL group; however, no significant difference was found compared with the healthy group. The expression level of PPP2R5C in the CML-CR group decreased significantly compared with that in the de novo CML group and was not significantly different from the level in the healthy group. By using different primer pairs that covered different exons, five transcript variants of PPP2R5C could be identified. All variants could be detected in healthy samples as well as in all the leukemia samples, and similar frequencies and distributions of PPP2R5C were indicated.

Conclusions: Overexpression of PPP2R5C in T-cell malignancy as well as in myeloid leukemia cells might relate to its proliferation and differentiation. Investigation of the effect of target inhibition of this gene might be beneficial to further characterization of molecular mechanisms and targeted therapy in leukemia.
\end{abstract}

Keywords: PPP2R5C leukemia, gene expression, transcript variant

\section{Background}

Molecular genetic aberrations could provide the basis for assays that can predict prognosis of individual patients as well as potential molecular targets for novel therapies [1-3]. The process of malignant transformation in leukemia is complex, and many factors such as abnormal gene expression and mutation, chromosomal aberrations, deregulation of various cellular signaling pathways, and deregulation of epigenetic regulation are involved in the development of leukemia [1]. Therefore, new data regarding molecular genetic aberrations in

\footnotetext{
* Correspondence: yangqiuli@hotmail.com

+ Contributed equally

'Institute of Hematology, Medical College, Jinan University, Guangzhou, P.R. China

Full list of author information is available at the end of the article
}

different types of leukemia are needed for further characterization.

PPP2R5C is one of the regulatory $\mathrm{B}$ subunits of protein phosphatase $2 \mathrm{~A}(\mathrm{PP} 2 \mathrm{~A})$, which is a major cellular serine/threonine phosphatase that affects the phosphorylation status of many proteins [4]. The PPP2R5C gene encodes five differentially spliced variants: B56 1 ,

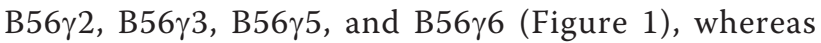
$\mathrm{B} 56 \gamma 4$ is identified only in mice. The functional $P P P 2 R 5 C$ gene locus resides at $14 \mathrm{q} 32.2$, whereas a nonfunctional B56 $\gamma 1$ pseudogene PPP2R5C is present at 3 p21.3 [4,5]. PPP2R5C plays a crucial role in cell proliferation, differentiation, and transformation, based on its induction of dephosphorylation of P53 at various residues [6]. It has been reported that the dynamic nuclear distribution of the B56 33 regulatory subunit controls
Ciomed Central

() 2011 Zheng et al; licensee BioMed Central Ltd. This is an Open Access article distributed under the terms of the Creative Commons Attribution License (http://creativecommons.org/licenses/by/2.0), which permits unrestricted use, distribution, and reproduction in any medium, provided the original work is properly cited. 


\section{PPP2R5C gene}

Transcript variant 1 (B56\%3)

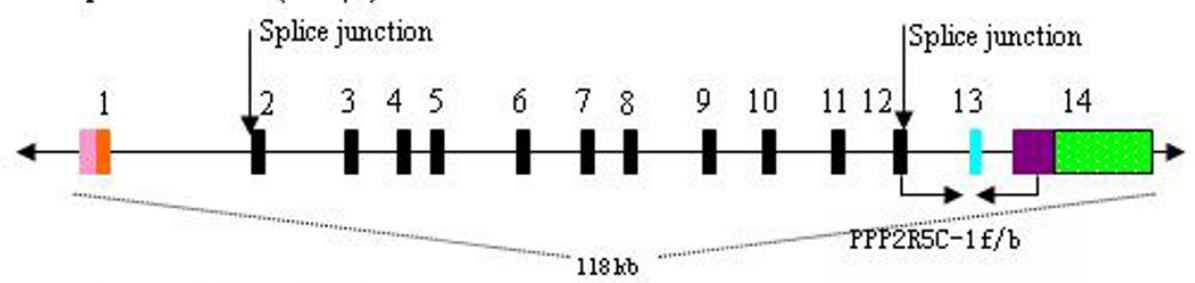

Transcript variant 2 (B56\%2)
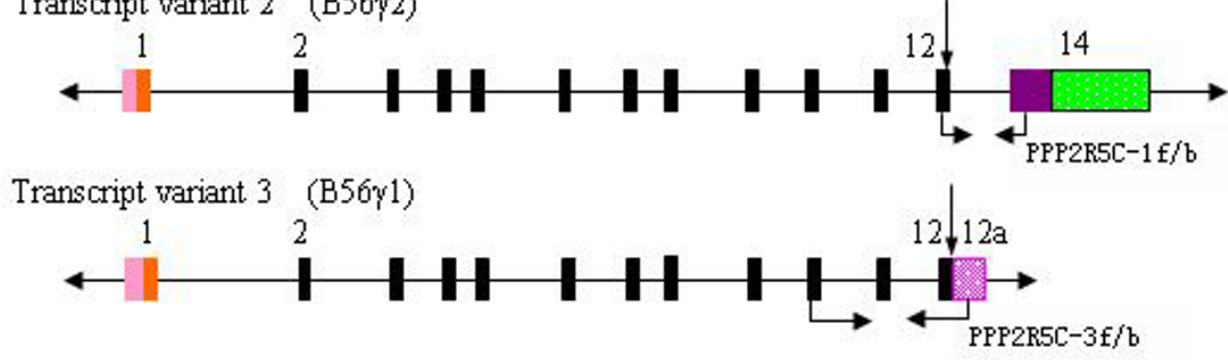

Transcript variant 5 (B56\%5)

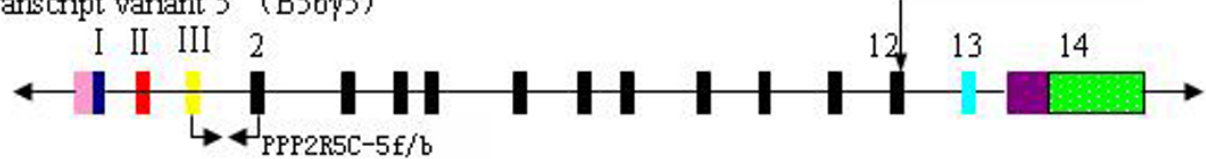

Transcript variant 6 (B56\%6)

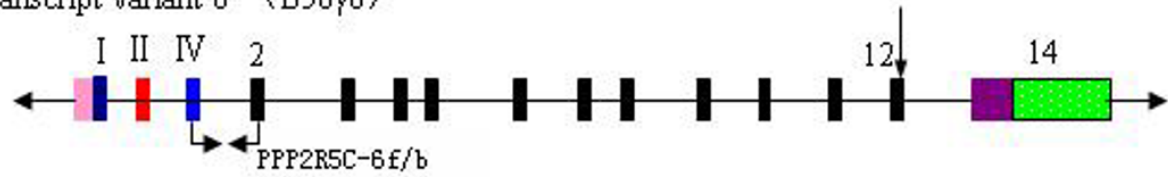

\section{PPP2RSC Pseudogene}

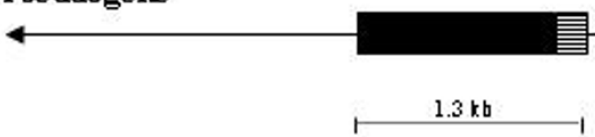

Figure 1 Genomic organization of PPP2R5C. The bars represent the exons, and the lines represent introns. The pink bars are the $5^{\prime}$ UTR, the black bars are exons that were identical in all five variants (exons 2-12), the colored bars represent specific exons in different variants, the $3^{\prime}$ UTR in different variants is shown with green bars (variants 1, 2, 5 and 6), horizontal-dash-filled pink bars (variant 3), and horizontal-line-filled bars (pseudogene). The coding sequence between exons 2 and 12 and the shown splice junction (downward arrow) were identical across the five splice variants. The location and direction of primers used for amplification of different variants are indicated by arrows [4].

nuclear PP2A activity and may be responsible for the tumor-suppression function of PP2A [5]. Recently, the alteration of the expression pattern of PPP $2 R 5 C$ associated with malignant transformation has been characterized in lung cancer; a PPP2R5C mutation, F395C, disrupts B56 $\gamma$-p53 interaction [7].

Based on our recent finding of a novel chromosomal translocation $\mathrm{t}(14 ; 14)(\mathrm{q} 11 ; \mathrm{q} 32)$ that was involved in a gene rearrangement from TRAJ7 to PPP $2 R 5 C$ in a patient with Sézary syndrome (data unpublished), in the present study, we further characterized the expression and distribution of five different transcript variants of the PPP2R5C gene in leukemia.

\section{Methods}

\section{Samples}

Seventy-seven newly diagnosed and untreated cases of leukemia, including 24 with acute myeloid leukemia (AML); 14 with chronic phase chronic myeloid leukemia (CML); 18 with T-cell acute lymphocytic leukemia/lymphoma (T-ALL); 12 with B-cell ALL (B-ALL); and nine with B-cell chronic lymphocytic leukemia (B-CLL). Twenty-six cases with leukemia in complete remission (CR) (nine with AML-CR, three with B-ALL-CR and 14 with CML-CR) were selected, along with 20 healthy individuals as controls. The samples were collected with informed consent. All procedures were conducted in 
accordance with the guidelines of the medical ethics committees of the Health Bureau of Guangdong Province, China. Human leukemia cell lines Hut-78, CCRF, Jurkat, Molt-3, Molt-4, K562, NB4, Raji and Daudi were used in the study. The RNA extraction and cDNA synthesis were performed according to the manufacturer's instructions.

\section{Real-time quantitative RT-PCR (qRT-PCR)}

Expression levels of PPP2R5C and the reference gene $\beta 2-M G$ were determined by SYBR Green I real-time PCR. PCR was performed as our previous description [8]. The $2^{(-\Delta \Delta C T)}$ method was used to present the data of the genes of interest relative to an internal control gene $[8,9]$. The sequences of primers used in qRT-PCR were PPP2R5C-for: 5'-GTAATAAAGCGGGCAGCAGG-3' and PPP2R5C-bac: 5'-CAAAGT CAAAGAGGACGCAACA-3' for PPP2R5C gene amplification, $\beta_{2}$ M-for: 5'-CAGCAAGG ACTGGTCTTTCTAT-3' and $\beta_{2}$ M-bac: 5'-GCGGCATCTTCAAACCTC-3' for $\beta_{2} \mathrm{M}$ gene amplification.

\section{Primer design and RT-PCR}

To amplify five transcript variants of PPP2R5C according the structure of the PPP2R5C gene (accession nos. NM_002719.3, NM_178586.2, NM_178587.2, NM_001161725.1 and NM_001161726.1) (Figure 1) [4], we designed 4-pair primers, which covered different exons (Table 1 ) and confirmed the transcript 3,5 , or 6 ; however, we were unable to distinguish between transcripts 1 and 5 or transcripts 2 and 6 (Table 2) when positive products were shown in the sample. RT-PCR was performed as in our previous study [10].

\section{Results and Discussion}

\section{Expression level of PPP2R5C in leukemia}

$P P P 2 R 5 C$ as a potential tumor suppressor plays a crucial role in cell proliferation and differentiation [4]. Based on our recent finding of a novel gene rearrangement from TRAJ7 to PPP2R5C, it could be interesting to analyze the expression features of PPP2R5C in hematological malignancies. In the present study, we analyzed the expression level of the PPP2R5C gene in leukemia samples. In comparison with healthy controls $(1.24 \pm 1.09)$, significantly higher expression of PPP $2 R 5 C$ was found in the AML $(2.06 \pm 0.85)(p=0.0076)$, CML $(6.78 \pm 2.75)$ $(p<0.0001), \mathrm{T}-\mathrm{ALL} / \mathrm{NHL}(3.73 \pm 3.66)(p=0.0062)$ and B-CLL $(2.21 \pm 1.22)(p=0.0417)$ groups (Figure 2$)$. A high tendency toward expression of PPP $2 R 5 C$ was detected in the B-ALL group $(1.39 \pm 1.31)$; however, the expression was not significantly different from that in the controls $(p=0.7089)$ (Figure 2). The expression level of PPP2R5C in the CML-CR group $(1.75 \pm 0.55)$ decreased significantly in comparison with the CML group $(p<0.0001)$, but showed no significant difference compared with the healthy group $(p=0.2895)$. Although the expression level of PPP2R5C gene decreased in the AML-CR $(1.53 \pm 0.60)$ and B-ALL groups $(0.54 \pm 0.27)$, there was no significant difference compared with the AML $(p=0.1100)$ and B-ALL groups $(p=0.2142)$ or with healthy controls. Overexpression of PPP2R5C was found in T-cell lines like Hut-78, CCRF, Jurkat, Molt-3 and Molt-4, and the expression level was 5-9 times higher than that from healthy $\mathrm{CD}^{+} \mathrm{T}$ cells (Figure 3A). Interesting, the tendency of the expression level of PPP2R5C in Raji, Daudi, NB4 and K562 cells was accordant to the results from primary leukemia cells (Figure $3 \mathrm{~B})$, which showed higher expression level of PPP2R5C in K562 (CML cell line), and lower expression level in B-cell lines (Raji and Daudi).

Mutation, deletion, or abnormal expression of tumorsuppressor genes is frequently identified in leukemia $[1,11,12]$. To the best of our knowledge, no study has been reported that examined the expression of PPP2R5C in leukemia, except for B-CLL. The present results identifying $P P P 2 R 5 C$ overexpression in most cases of leukemia might have a similar significance in cell transformation. Definitive results for the association of the expression level of PPP2R5C with disease progression and outcome could be demonstrated by a

Table 1 Information on primers used in RT-PCR for PPP2R5C segments amplification

\begin{tabular}{|c|c|c|c|}
\hline primer & sequence & Location & function \\
\hline PPP2R5C-1f & 5'-TGAAAGAACGGGAAGAAGCAT - 3' & 1407 bp (12 exon) & Sense primer \\
\hline PPP2R5C-1b & 5'-TGATTGGTATGGCACAGGAAG - 3' & 1801 bp (14 exon) & Antisense primer \\
\hline PPP2R5C-3f & 5'-CAGTGACAACGCAGCGAAGAT - 3' & 1216 bp (10 exon) & Sense primer \\
\hline PPP2R5C-3b & 5'-ATAAAAACATTCAAGTAACCCTGG-3' & 1520 bp (12a exon) & Antisense primer \\
\hline PPP2R5C-5f & 5'-TCCACTTCTTCCTGAGTTGCTG-3' & 230 bp (III exon) & Sense primer \\
\hline PPP2R5C-5b & 5'-CTTCTGGGTAAATAGGCTCTGT-3' & 472 bp (2 exon) & Antisense primer \\
\hline PPP2R5C-6f & 5'-AGCCTTGTTGCTGTCCCGTCT - 3' & 210 bp (IV exon) & Sense primer \\
\hline PPP2R5C-6b & 5'-GTCAAAGAGGACGCAACACTG - 3' & 423 bp (2 exon) & Antisense primer \\
\hline
\end{tabular}


Table 2 Amplified PCR products using different primer pairs

\begin{tabular}{|c|c|c|c|c|c|}
\hline Primer pairs & $\begin{array}{c}\text { Variant } 1 \\
(\mathrm{~B} 56 \gamma 3)\end{array}$ & $\begin{array}{c}\text { Variant } 2 \\
\text { (B56 } 2 \text { 2) }\end{array}$ & $\begin{array}{c}\text { Variant } 3 \\
\text { (B56y1) }\end{array}$ & $\begin{array}{c}\text { Variant } 5 \\
(\mathrm{~B} 56 \gamma 5)\end{array}$ & $\begin{array}{c}\text { Variant } 6 \\
\text { (B56y6) }\end{array}$ \\
\hline PPP2R5C1f/PPP2R5C1b & $\begin{array}{c}+ \\
12+13+14 \text { exons } \\
(394 \text { bp) }\end{array}$ & $\stackrel{+}{+}$ & - & $\begin{array}{c}+ \\
12+13+14 \text { exons } \\
(394 \text { bp) }\end{array}$ & $\stackrel{+}{+}$ \\
\hline PPP2R5C3f/PPP2R5C3b & - & - & $\begin{array}{c}\stackrel{+}{+} 10+12^{+}+12 \mathrm{a} \text { exons } \\
(304 \mathrm{bp})\end{array}$ & - & - \\
\hline PPP2R5C5f/PPP2R5C5b & - & - & - & $\begin{array}{c}+ \\
\text { III }+2 \text { exons } \\
(242 \text { bp) }\end{array}$ & - \\
\hline PPP2R5C6f/PPP2R5C6b & - & - & - & - & $\begin{array}{c}\quad+ \\
\text { IV }+2 \text { exons } \\
(213 \text { bp) }\end{array}$ \\
\hline
\end{tabular}

comparison of the data for de novo CML and CML in complete remission. In contrast, Fält et al. have described that downregulated $P P P 2 R 5 C$ is related to progressive B-CLL by using the Affymetrix GeneChip technique, and they have concluded that PPP2R5C could be a marker of progressive disease in B-CLL. They have compared expression of PPP2R5C between stable and progressive B-CLL, but not with a healthy control group [13]. In the present study, we found that expression of PPP2R5C was significantly increased in the B-CLL group, similar to AML, CML, and T-ALL; however, we were unable to collect samples from patients in complete remission and from those with progressive disease to compare the expression level and evaluate the significance of PPP $2 R 5 C$ detection in $\mathrm{B}$ CLL. Further investigation is needed. Unlike most

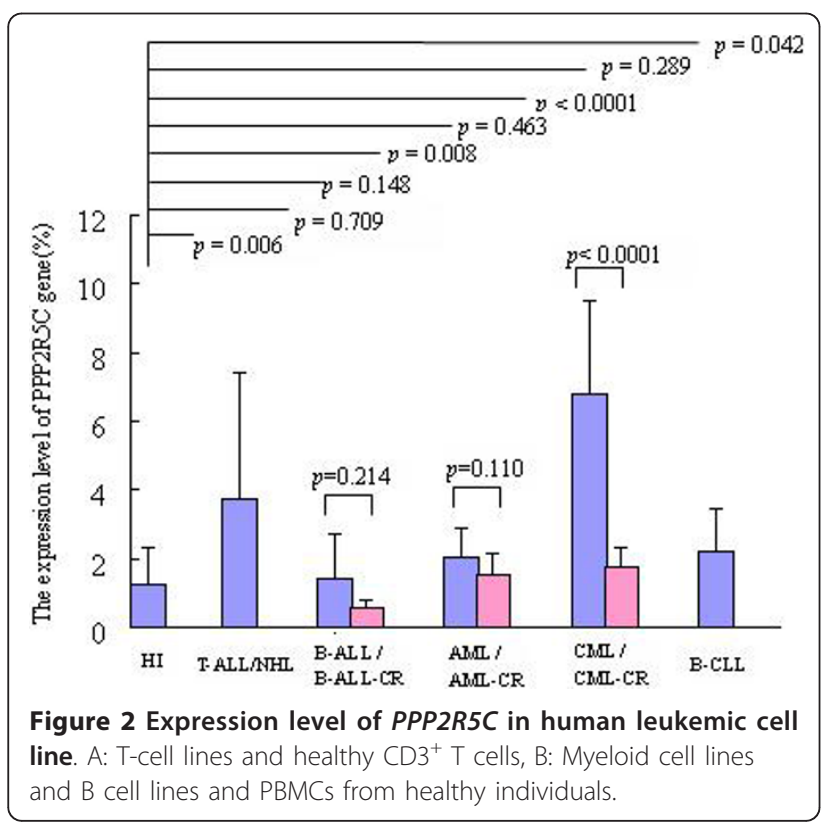

leukemia cases, the expression level of PPP2R5C in the B-ALL group, as well as in B-cell lines did not differ significantly from that in the controls, but it remains unknown whether this was due to the limited number of samples or whether it reflects a true feature of $P P P 2 R 5 C$ in B-ALL.

\section{Distribution of PPP2R5C transcript variants in healthy individuals and leukemia}

It has been reported that five transcript variants of PPP2R5C might contribute to the specificity of PP2A [4]. However, little is known about the distribution of different variants in different organs, tissues, and cells, as well as in leukemia cells. Based on the structure of the PPP2R5C gene reported from Genbank and previous studies [4], we drew a schematic diagram of the genomic organization of $P P P 2 R 5 C$ with five transcript variants (Figure 1), designed four primer pairs to amplify different exons, and tried to identify different variants in the same sample. By using PPP2R5C-1f/PPP2R5C-1b, which covered exons 12-14, two expected PCR products were detected. The small one comprised 277 bp containing exon 12 and 14 segments (corresponding to B56 2 or B56 6 ), and the large one comprised $394 \mathrm{bp}$ containing exon 12,13, and 14 segments (correspond-

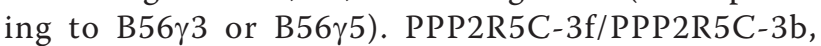
PPP2R5C-5f/PPP2R5C-5b, and PPP2R5C-6f/PPP2R5C$6 \mathrm{~b}$ primer pairs covered exons 10 to $12 \mathrm{a}$, exon III to 2 , and exon IV to 2, respectively, and the expected PCR products were 304, 242, and $213 \mathrm{bp}$ (corresponding to transcript $\mathrm{B} 56 \gamma 1, \mathrm{~B} 56 \gamma 5$, and $\mathrm{B} 56 \gamma 6$ respectively) (Table 2 Figure 4). According the structure of transcript variants of $P P P 2 R 5 C$ gene and the size of the amplicons, we confirmed the B56 $\gamma 1$, B $56 \gamma 5$, and B $56 \gamma 6$ variants. However, using the designed primer pairs, we were unable to distinguish between transcripts 1 and 5 or between transcripts 2 and 6 because PCR products of the same size were amplified by using the PPP2R5C-1f/ 


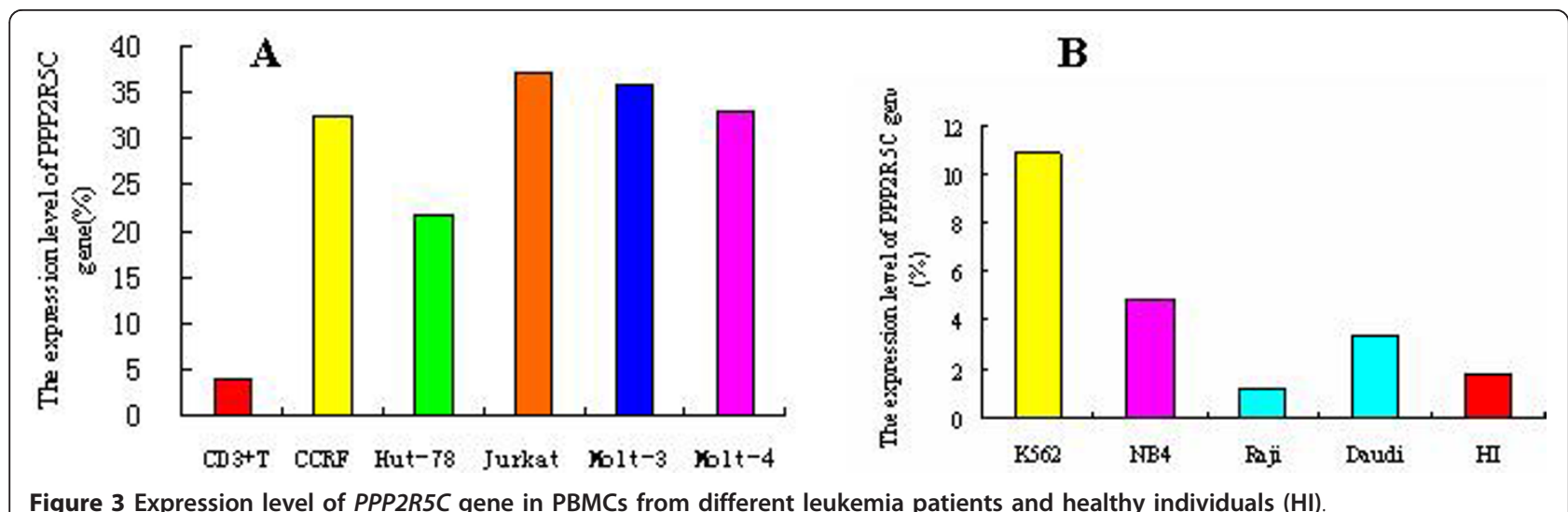

PPP2R5C-1b, and it could not distinguish these variants using different primer pair combinations, except for

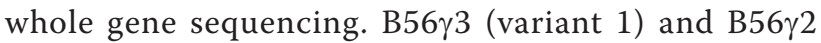
(variant 2) are the frequency variants; therefore, it is thought that they might be expressed when positive PCR products are found. More importantly, using the present methods, we confirmed the expression of B56 75 and B56 6 , which have been newly identified. Therefore, it could be concluded that all variants can be detected in healthy as well as leukemia samples with a similar frequency and distribution of PPP2R5C.

In conclusion, to the best of our knowledge, this is the first description of the expression level of the PPP2R5C gene as well as the distribution of PPP2R5C transcript variants in $\mathrm{PBMCs}$ from different types of leukemia. Overexpression of PPP2R5C is a common feature in most types of leukemia; thus, the change in expression pattern might influence the activity of PP2A and could be related to abnormal cell proliferation, differentiation, and transformation. Further research on the

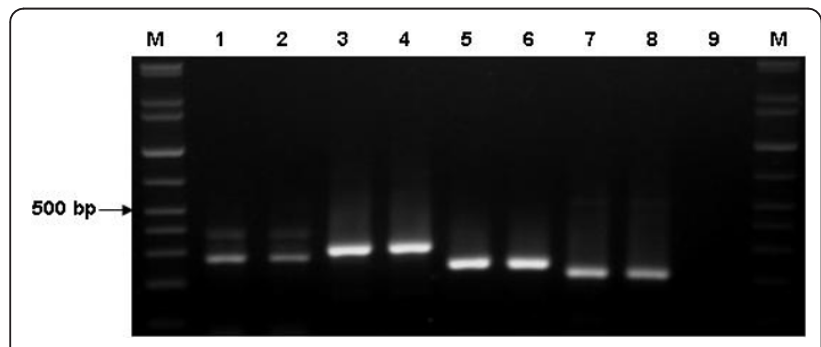

Figure 4 Results of PCR amplification for PPP2R5C gene using different primer pairs. Lane M: 100-bp DNA ladder; 1 and 2: amplicom using PPP2R5C1f-/PPP2R5C1b primers; small products were 277 bp (12 + 14 exons) and large products were 394 bp (12 + $13+14$ exons); 3 and 4: amplicom using PPP2R5C3f/PPP2R5C3b primers, the product was $304 \mathrm{bp}(10+11+12+12 \mathrm{a}$ exons $) ; 5$ and 6: amplicom using PPP2R5C5f/PPP2R5C5b primers, the product was 242 bp (III +2 exons); 7 and 8: ampilcom using PPP2R5C6f/ PPP2R5C6b primers, the product was 213 bp (IV + 2 exons); 9: negative control. downregulation of PPP2R5C in leukemia cells is needed to investigate its biological function.

\section{Acknowledgements}

This work was supported by Grants from National Natural Science Foundation of China (no. 30871091), the Fundamental Research Funds for the Central Universities (No. 21610604) and Science and Technology Innovation Key Project of Guangdong Higher Education Institutes (kjcxzd1013).

\section{Author details}

${ }^{1}$ Institute of Hematology, Medical College, Jinan University, Guangzhou, P.R China. ${ }^{2}$ Key Laboratory for Regenerative Medicine of Ministry of Education, Jinan University, Guangzhou, P.R. China. ${ }^{3}$ Department of Hematology, Guangdong General Hospital (Guangdong Academy of Medical Sciences), Guangzhou, P.R. China.

Authors' contributions

YQL contributed to concept development and study design. HTZ performed the real-time PCR, YC performed the RT-PCR, SHC and YZN prepared RNA and CDNA, LJY and BL prepared the PBMCs and collected the clinical data. $Y H L, S X G$ and $X D$ were responsible of the patient's treatment and carried out acquisition of clinical data. YQL, HTZ and YC coordinated the study and helped to draft the manuscript. All authors read and approved the final manuscript.

\section{Competing interests}

No potential conflicts of interest and financial disclosure statements except for grants mentioned in the acknowledgements.

Received: 22 March 2011 Accepted: 6 May 2011 Published: 6 May 2011

\section{References}

1. Bacher U, Kohlmann A, Haferlach T: Gene expression profiling for diagnosis and therapy in acute leukaemia and other haematologic malignancies. Cancer Treat Rev 2010, 36:637-646.

2. Braoudaki M, Papathanassiou C, Katsibardi K, Tourkadoni N, Karamolegou K, Tzortzatou-Stathopoulou F: The frequency of NPM1 mutations in childhood acute myeloid leukaemia. J Hematol Oncol 2010, 3:41.

3. Zhu X, Ma Y, Liu D: Novel agents and regimens for acute myeloid leukemia: 2009 ASH annual meeting highlights. J Hematol Oncol 2010, 3:17.

4. Muneer S, Ramalingam V, Wyatt R, Schultz RA, Minna JD, Kamibayashi C: Genomic organization and mapping of the gene encoding the PP2A B56gamma regulatory subunit. Genomics 2002, 79:344-348.

5. Lee TY, Lai TY, Lin SC, Wu CW, Ni IF, Yang YS, Hung LY, Law BK, Chiang CW: The B56gamma3 regulatory subunit of protein phosphatase 2A (PP2A) regulates $S$ phase-specific nuclear accumulation of PP2A and the $G 1$ to S transition. J Biol Chem 2010, 285:21567-21580. 
6. Shouse GP, Cai X, Liu X: Serine 15 phosphorylation of p53 directs its interaction with B56gamma and the tumor suppressor activity of B56gamma-specific protein phosphatase 2A. Mol Cell Biol 2008, 28:448-456.

7. Shouse GP, Nobumori Y, Liu X: A B56gamma mutation in lung cancer disrupts the p53-dependent tumor-suppressor function of protein phosphatase 2A. Oncogene 2010, 29:3933-3941.

8. Huang X, Chen S, Shen Q, Yang L, Li B, Zhong L, Geng S, Du X, Li Y: Analysis of the expression pattern of the $B C L 11 B$ gene and its relatives in patients with T-cell acute lymphoblastic leukemia. J Hematol Oncol 2010, 3:44.

9. Livak KJ, Schmittgen TD: Analysis of relative gene expression data using real-time quantitative PCR and the 2(-Delta Delta $C(T)$ ) Method. Methods 2001, 25:402-408.

10. Wang L, Zhu K, Zha X, Chen S, Yang L, Chen S, Li Y: Evolution of T-cell clonality in a patient with $\mathrm{Ph}$-negative acute lymphocytic leukemia occurring after interferon and imatinib therapy for Ph-positive chronic myeloid leukemia. J Hematol Oncol 2010, 3:14.

11. Oshiro A, Tagawa H, Ohshima K, Karube K, Uike N, Tashiro Y, Utsunomiya A, Masuda M, Takasu N, Nakamura S, Morishima Y, Seto M: Identification of subtype-specific genomic alterations in aggressive adult T-cell leukemia/ lymphoma. Blood 2006, 107:4500-4507.

12. Hämäläinen $M M$, Kairisto $V$, Juvonen $V$, Johansson J, Aurén J, Kohonen $K$, Remes K, Salmi TT, Helenius H, Pelliniemi TT: Wilms tumour gene 1 overexpression in bone marrow as a marker for minimal residual disease in acute myeloid leukaemia. Eur J Haematol 2008, 80:201-207.

13. Fält S, Merup M, Gahrton G, Lambert B, Wennborg A: Identification of progression markers in B-CLL by gene expression profiling. Exp Hematol 2005, 33:883-893.

doi:10.1186/1756-8722-4-21

Cite this article as: Zheng et al: Expression and distribution of PPP2R5C gene in leukemia. Journal of Hematology \& Oncology 2011 4:21.

\section{Submit your next manuscript to BioMed Central and take full advantage of:}

- Convenient online submission

- Thorough peer review

- No space constraints or color figure charges

- Immediate publication on acceptance

- Inclusion in PubMed, CAS, Scopus and Google Scholar

- Research which is freely available for redistribution

Submit your manuscript at www.biomedcentral.com/submit
Biomed Central 